\title{
Análise florística e fitogeografia das samambaias e licófitas de um fragmento de Mata Atlântica na Serra da Jibóia, Santa Teresinha, Bahia, Brasil ${ }^{1}$
}

Floristic analysis and phytogeography of ferns and lycophytes from an Atlantic Forest remnant in the Serra da Jibóia, Santa Teresinha, Bahia, Brazil

\author{
Taís Soares Macedo ${ }^{2,4}$, Aristóteles Góes Neto ${ }^{2}$ \& Fabiana Regina Nonato ${ }^{3}$
}

\begin{abstract}
Resumo
O trabalho apresenta espécies de samambaias e licófitas de um fragmento de Mata Atlântica, inserido na região de semi-árido, localizado na Serra da Jibóia, Município de Santa Teresinha, Bahia, Brasil e a análise das relações florísticas com outras formações vegetais do país, contribuindo para ampliar as informações sobre a flora vascular do estado, bem como do bioma Mata Atlântica. São apresentadas 97 espécies, em 51 gêneros e 22 famílias. As famílias mais representativas foram Dryopteridaceae (17 spp.), Polypodiaceae (16 spp.), Pteridaceae (12 spp.) e Hymenophyllaceae (10 spp.). A maioria das espécies ocorre como epífita (48\%), 38\% terrestre, 3\% rupícola, 3\% como hemiepífita e 8\% com mais de um tipo de hábito. A análise de similaridade mostra que a flora de samambaias e licófitas da região está relacionada com o Sul da Bahia e Sudeste do Brasil, sugerindo que a área de estudo apresenta condições ambientais semelhantes às áreas relacionadas e reforçando as singularidades florísticas da região.
\end{abstract}

Palavras-chave: fitogeografia, Mata Atlântica, samambaias, licófitas.

\begin{abstract}
The work presents species of ferns and lycophytes from a remnant of Atlantic Forest located in a semi-arid region, in the Serra da Jibóia, municipality of Santa Teresinha, State of Bahia, Brazil. We also analyzed the floristic similarities of this area with other regions in Brazil. In total, 97 species were presented, representing 51 genera and 22 families. The most representative families were Dryopteridaceae (17 spp.), Polypodiaceae (16 spp.), Pteridaceae (12 spp.), and Hymenophyllaceae (10 spp.). Most species occur as epiphytes (48\%), whereas $38 \%$ are terrestrial, $3 \%$ are lythophyte, $3 \%$ are hemiepiphyte and $8 \%$ present more than one type of habit. The similarity analysis showed that the flora of ferns and lycophytes from Serra da Jibóia is related to the Southern Bahia State and Southeastern Brazil areas, suggesting similar environmental conditions among those areas.
\end{abstract}

Key words: phytogeography, Atlantic Forest, ferns, lycophytes.

\section{Introdução}

Considerada uma das regiões mais ricas em diversidade biológica e também mais ameaçadas do planeta, a Mata Atlântica figura entre os 34 hotspots mundiais (Mittermeier et al. 2004). Apesar de ter perdido mais de $93 \%$ de sua cobertura original, o bioma resguarda em torno de 20.000 espécies de plantas vasculares, das quais cerca de 8.000 são endêmicas (Myers et al. 2000), apresentando importantes variações florísticas e estruturais ao longo de sua distribuição geográfica (Oliveira-Filho \& Fontes 2000). Segundo Fernandes (2000), as variações fisionômicas apresentadas pela vegetação atlântica estão relacionadas com a dinâmica sucessional das associações florísticas a partir do Terciário. A existência de numerosos representantes das pteridófitas e gimnospermas, nesta vegetação,

\footnotetext{
${ }^{1}$ Parte da dissertação de Mestrado da primeira autora.

${ }^{2}$ Universidade Estadual de Feira de Santana-UEFS, Depto. Ciências Biológicas, Programa de Pós-Graduação em Botânica, Av. Transnordestina s/n, 44036-900, Feira de Santana, BA, Brasil.

${ }^{3}$ Fundação Oswaldo Cruz-FIOCRUZ, Centro de Pesquisas Gonçalo Moniz, R. Waldemar Falcão 121, 40296-710, Salvador, BA, Brasil.

${ }^{4}$ Autora para correspondência: taismacedo10@yahoo.com.br
} 
empresta um significado singular no contexto fitogeográfico brasileiro.

De acordo com Mori et al. (1983), a Mata Atlântica do estado da Bahia apresenta uma expressiva diversidade florística. Mais recentemente, a Conservação Internacional do Brasil et al. (2000), ao indicar as 147 áreas prioritárias para conservação da flora do bioma, ressalta que $48 \%$ destas encontram-se na região Nordeste, principalmente nos estados da Bahia, Ceará, Alagoas e Pernambuco. Além disso, enfatiza que os inventários biológicos e ações relacionadas às unidades de conservação são recomendações pertinentes para as áreas prioritárias, pois apesar de possuir mais de 300 unidades de conservação, estas não contemplam as diferentes sub-regiões geográficas presentes no bioma e os remanescentes existentes têm sido pouco estudados, resultando em grandes lacunas no conhecimento destas.

Neste sentido, uma das lacunas no conhecimento do bioma diz respeito às samambaias e licófitas da Mata Atlântica baiana. O grupo tem sido citado em trabalhos mais amplos, envolvendo a flora vascular como um todo. Entre os levantamentos específicos para o grupo, destacamse os realizados na Chapada Diamantina por Edwards (1995), Øllgaard (1995), Prado (1995), Barros (1998), Barros \& Araújo (2000), Prado (2003), Nonato (2005) e Ferreira et al. (2012), sendo estes, trabalhos que abordam apenas a flora em questão. Apesar das samambaias e licófitas apresentarem-se distribuídas principalmente nas áreas de Floresta Atlântica do Brasil, como discutido em Labiak \& Prado (1998) e Salino \& Almeida (2009), trabalhos realizados na Bahia, no referido bioma, ainda são incipientes (Noblick \& Britto 1984; Paciência \& Prado 2004; 2005; Matos et al. 2010 e Macedo et al. 2012).

Recentemente, realizou-se o estudo da flora das samambaias e licófitas de um fragmento de Mata Atlântica no estado da Bahia, ressaltando a diversidade de espécies neste bioma com novos registros para o Estado, bem como para a região Nordeste (Macedo et al. 2012). Desta forma, o presente trabalho objetivou analisar as relações florísticas das espécies de samambaias e licófitas da referida área, a qual encontra-se inserida na região de semi-árido, localizada na Serra da Jibóia, Município de Santa Teresinha, Bahia, Brasil, comparando com outras formações vegetais do país.

\section{Material e Métodos}

Área de estudo

A Serra da Jibóia compreende um maciço de morros localizado no Município de Santa Teresinha, Estado da Bahia, próximo ao limite do Município de Castro Alves e Elísio Medrado, na porção sul do Recôncavo Baiano (Fig. 1). O fragmento estudado encontra-se, mais precisamente, no Morro da Pioneira, extremo norte da Serra, a uma latitude aproximada de $12^{\circ} 51^{\prime} \mathrm{S}$ e longitude de $39^{\circ} 28^{\prime} \mathrm{W}$. A altitude máxima alcança $800 \mathrm{~m}$.

A região apresenta certas peculiaridades, abrigando grande diversidade de tipos vegetacionais ocorrendo, no lado oeste da serra, extensas áreas de caatinga, Mata Ombrófila Densa ao leste e uma vegetação de campo rupestre no topo. Constitui-se um dos pontos mais oeste da mata atlântica baiana e uma das matas úmidas de encosta situada mais ao norte do estado (Juncá 2006). O clima da região é tropical semi-úmido com temperatura média anual de $22^{\circ} \mathrm{C}$, variando em função da altitude. $\mathrm{O}$ índice pluviométrico anual é de aproximadamente 1200 mm (Sandes 2003).

A Serra da Jibóia é considerada uma das 182 áreas prioritárias para conservação da biodiversidade da Mata Atlântica brasileira, sendo uma das 99 áreas de extrema importância biológica, segundo a Conservação Internacional do Brasil et al. (2000).

\section{Municípios do Recôncavo do Sul}

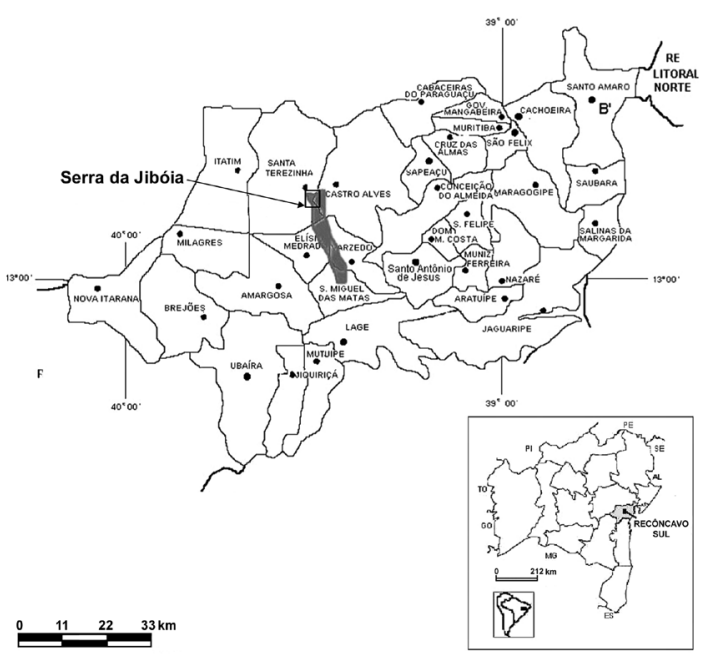

Figura 1 - Mapa com a localização da Serra da Jibóia (em destaque) e indicação do Morro da Pioneira ( $\square$ ). Fonte: Adaptado de Tomasoni (2000).

Figure 1 - Map showing the Serra da Jibóia (featured) and indication of the Morro da Pioneira ( $\square$ ). Source: Adapted from Tomasoni (2000). 
Análise florística e fitogeografia

Foram realizadas excursões de coletas mensais à área de estudo, durante os anos de 2009 e 2010. O material botânico foi coletado e herborizado de acordo com as técnicas descritas por Windisch (1992) e identificado a partir de bibliografia específica. Também foram incluídos materiais das coleções dos herbários ALCB, HRB e HUEFS (siglas segundo Thiers, 2010). Todo material coletado encontra-se depositado no Herbário da Universidade Estadual de Feira de Santana - HUEFS.

A classificação adotada para as samambaias está de acordo com Smith et al. (2006; 2008) e para as licófitas segue Kramer \& Green (1990). A abreviação dos nomes dos autores segue Pichi-Sermolli (1996).

Os dados a respeito da distribuição geográfica foram baseados em bibliografia especializada e levantamento das coleções oriundas dos Herbários HUEFS, HRB, ALCB, UFP, IPA, PEUFR e os padrões adotados baseiam-se em macro-regiões como estabelecido por Lima et al. (1997). A caracterização adotada para os tipos de hábito foram: terrestre, epífita, hemiepífita e rupícola.
Similaridade florística

Foi realizada a comparação florística a fim de se verificar a similaridade entre a área de estudo e outras 26 áreas, correspondendo a diferentes formações vegetacionais do país (Tab. 1). As fisionomias foram classificadas de acordo com a definição já apresentada nos trabalhos escolhidos para a análise, seguindo basicamente a classificação de Veloso et al. (1991). Elaborou-se uma matriz de dados binários (presença/ ausência) das espécies. Espécies indeterminadas foram desconsideradas e todas as outras tiveram os seus binômios checados e atualizados segundo a classificação adotada, quando necessário.

As relações de afinidade foram determinadas pelo Índice de Similaridade de Sørensen. Neste índice os valores variam de 0 a 1 , sendo que valores acima de 0,5 indicam uma elevada similaridade (Margurran 1988). Para interpretar a similaridade entre as áreas foi realizada a análise de agrupamento pelo método média de grupo (UPGMA) e tais relações foram representadas através de dendrograma. As análises foram realizadas utilizando-se o programa PAST (Hammer et al. 2001).

Tabela 1 - Relação das áreas utilizadas na análise de similaridade florística. FES = Floresta Estacional Semidecidual; FED = Floresta Estacional Decidual; FOD = Floresta Ombrófila Densa; FOM=Floresta Ombrófila Mista; SAV = Savana Table 1 - List of the areas used in the analysis of floristic similarity. FES = Semideciduous Seasonal Forest; FED = Deciduous Seasonal Forest; FOD = Dense Ombrophilous Forest; FOM = Mixed Ombrophilous Forest; SAV = Savanna.

\begin{tabular}{|c|c|c|c|c|c|}
\hline $\begin{array}{l}\text { Código no } \\
\text { dendrograma }\end{array}$ & Localidade & Fisionomia & Coordenadas & Espécies & Referências \\
\hline DuckeAM & Reserva Ducke, AM & FOD Amazônica & $3^{\circ} 06^{\prime} \mathrm{S}$ e $60^{\circ} 01^{\prime} \mathrm{W}$ & 83 & Costa et al. (1999) \\
\hline RNegroAM & Santa Isabel do Rio Negro, AM & FOD Amazônica & $0^{\circ} 28^{\prime} \mathrm{S}$ e $65^{\circ} 32^{\prime} \mathrm{W}$ & 48 & Freitas \& Prado (2005) \\
\hline UatumãAM & REBIO Uatumã, AM & FOD Amazônica & $2^{\circ} 34^{\prime} \mathrm{S}$ e $57^{\circ} 52^{\prime} \mathrm{W}$ & 123 & Zuquim et al. (2008) \\
\hline IlhaMosqPA & Ilha de Mosqueiro, PA & FOD Amazônica & $1^{\circ} 04^{\prime} \mathrm{S}$ e $48^{\circ} 28^{\prime} \mathrm{W}$ & 65 & Costa \& Pietrobom (2007) \\
\hline PqBelémPA & Pq. Amb. de Belém, PA & FOD Amazônica & $1^{\circ} 23^{\prime}$ 'S e $48^{\circ} 26^{\prime} \mathrm{W}$ & 49 & Costa et al. (2006) \\
\hline BonitoPE & Bonito, PE & FES & $8^{\circ} 28^{\prime} \mathrm{S}$ e $35^{\circ} 43^{\prime} \mathrm{W}$ & 93 & Santiago et al. (2004) \\
\hline CaruaruPE & $\begin{array}{l}\text { Pq. Est. João Vasconcelos Sobrinho, } \\
\text { Caruaru, PE }\end{array}$ & FES & $8^{\circ} 16^{\prime} \mathrm{S}$ e $35^{\circ} 58^{\prime} \mathrm{W}$ & 74 & Xavier \& Barros (2005) \\
\hline TimbaúbPE & Engenho Água Azul, Timbaúba, PE & FES & $7^{\circ} 30^{\prime}$ S e $35^{\circ} 19^{\prime} \mathrm{W}$ & 85 & Pietrobom \& Barros (2007) \\
\hline JosLajeAL & São José da Laje, AL & FES & $9^{\circ} 00^{\prime} \mathrm{S}$ e $36^{\circ} 03^{\prime} \mathrm{W}$ & 76 & Pietrobom \& Barros (2006) \\
\hline SBonitaBA & RPPN Serra Bonita, Camacan, BA & FOD & $15^{\circ} 25^{\prime} \mathrm{S}$ e $39^{\circ} 29^{\prime} \mathrm{W}$ & 182 & Matos et al. (2010) \\
\hline SJibóiaBA & Serra da Jibóia, Sta Teresinha, BA & FOD & $12^{\circ} 51^{\prime} \mathrm{S}$ e $39^{\circ} 28^{\prime} \mathrm{W}$ & 97 & Presente estudo \\
\hline UnaBA & REBIO Una, BA & FOD & $15^{\circ} 17^{\prime} \mathrm{S}$ e $39^{\circ} 04^{\prime} \mathrm{W}$ & 60 & Paciência \& Prado (2005) \\
\hline SRFrancMT & Serra de Ricardo Franco, MT & FES, FOD & $15^{\circ} 10^{\prime} \mathrm{S}$ e $60^{\circ} 00^{\prime} \mathrm{W}$ & 111 & Windisch \& Tryon (2001) \\
\hline PantanalMS & Pantanal, MS & FES, FED, SAV & $19^{\circ} 00^{\prime} \mathrm{S}$ e $57^{\circ} 39^{\prime} \mathrm{W}$ & 114 & Assis (2007) \\
\hline CaratingMG & Est. Biol. de Caratinga, MG & FES & $19^{\circ} 48^{\prime} \mathrm{S}$ e $41^{\circ} 48^{\prime} \mathrm{W}$ & 103 & Melo \& Salino (2002) \\
\hline FerDiasMG & APA Fernão Dias, MG & FES, FOD, FOM & $22^{\circ} 45^{\prime} \mathrm{S}$ e $46^{\circ} 08^{\prime} \mathrm{W}$ & 173 & Melo \& Salino (2007) \\
\hline ItalacomiMG & Pq. Est. do Italacomi, MG & FES & $20^{\circ} 30^{\prime} \mathrm{S}$ e $43^{\circ} 22^{\prime} \mathrm{W}$ & 170 & Rolim (2007) \\
\hline PRDoceMG & Pq. Est. do Rio Doce, $\mathrm{MG}$ & FES & $19^{\circ} 30^{\prime} \mathrm{S}$ e $42^{\circ} 28^{\prime} \mathrm{W}$ & 123 & Melo \& Salino (2002) \\
\hline
\end{tabular}




\begin{tabular}{|c|c|c|c|c|c|}
\hline $\begin{array}{l}\text { Código no } \\
\text { dendrograma }\end{array}$ & Localidade & Fisionomia & Coordenadas & Espécies & Referências \\
\hline MacCimaRJ & $\begin{array}{l}\text { Res. Ecol. Macaé de Cima } \\
\text { Nova Friburgo, RJ }\end{array}$ & FOD & $22^{\circ} 16^{\prime} \mathrm{S}$ e $42^{\circ} 31^{\prime} \mathrm{W}$ & 81 & Lima et al. (2001) \\
\hline RPedrasRJ & $\begin{array}{l}\text { RPPN Rio das Pedras, } \\
\text { Mangaratiba, RJ }\end{array}$ & FOD & $22^{\circ} 57^{\prime} \mathrm{S}$ e $44^{\circ} 02^{\prime} \mathrm{W}$ & 117 & Mynssen \& Windisch (2004) \\
\hline IlhaAnchSP & Pq. Est. Ilha Anchieta, Ubatuba, SP & FOD & $23^{\circ} 31^{\prime} \mathrm{S}$ e $45^{\circ} 02^{\prime} \mathrm{W}$ & 94 & Athayde Filho et al. (2003) \\
\hline JacupirSP & Pq. Est. do Jacupiranga, SP & FOD & $24^{\circ} 45^{\prime} \mathrm{S}$ e $48^{\circ} 30^{\prime} \mathrm{W}$ & 212 & Salino \& Almeida (2008) \\
\hline KlabinPR & $\begin{array}{l}\text { Pq. Est. da Klabin, } \\
\text { Telêmaco Borba, PR }\end{array}$ & EST, FOM, FES & $24^{\circ} 19^{\prime} \mathrm{S}$ e $50^{\circ} 36^{\prime} \mathrm{W}$ & 121 & Sakagami (2006) \\
\hline MarumbiPR & $\begin{array}{l}\text { Pq. Est. Pico do Marumbi, } \\
\text { Morretes, PR }\end{array}$ & FOM & $25^{\circ} 28^{\prime} \mathrm{S}$ e $48^{\circ} 50^{\prime} \mathrm{W}$ & 81 & Dittrich et al. (2005) \\
\hline VilaVelhaPR & Pq. Est. Vila Velha, Ponta Gossa, PR & EST, FOM & $25^{\circ} 05^{\prime} \mathrm{S}$ e $50^{\circ} 09^{\prime} \mathrm{W}$ & 152 & Schwartsburd \& Labiak (2007) \\
\hline TeutôniaRS & Morro da Harmonia, Teutônia, RS & FED, FOM & - & 71 & Steffens \& Windisch (2007) \\
\hline FlCanelaRS & Floresta Nac. de Canela, RS & FOM & $29^{\circ} 19^{\prime}$ 'S e $50^{\circ} 48^{\prime} \mathrm{W}$ & 58 & Schmitt et al. (2006) \\
\hline
\end{tabular}

\section{Resultados e Discussão}

Análise florística e fitogeografia

$\mathrm{Na}$ área de estudo ocorrem 97 espécies, distribuídas em 51 gêneros e 22 famílias (Macedo et al. 2012) (Tab. 2). O número de samambaias e licófitas citado corresponde a $26 \%$ da flora vascular do fragmento estudado, se levarmos em consideração as 269 espécies de angiospermas registradas em Sobrinho \& Queiroz (2005).

Com relação ao hábito, a maioria das espécies (48\%) foi relatada como epífita, 38\% ocorrem como terrestre, $3 \%$ como rupícola, $3 \%$ como hemiepífita e $8 \%$ com mais de um tipo de hábito (Tab. 2). Na maior parte dos levantamentos realizados no Brasil, envolvendo samambaias e licófitas, o hábito terrestre se destaca entre as espécies. Entretanto, a riqueza de epífitas no estudo pode ser equiparada com outros trabalhos realizados no país, tais como Sylvestre (1997), Dittrich et al. (2005) e Paciência (2008), em que a prevalência do hábito é relacionada à elevada umidade nos ambientes estudados (Kornaš 1977), bem como a sua capacidade de sobrevivência em ambientes submetidos a grandes variações abióticas (Moran et al. 2003). Além de crescerem em substratos que apresentam menor disponibilidade hídrica e de nutrientes, as epífitas ainda estão susceptíveis a grandes variações sazonais e diárias de umidade (Benzing 1990; Mehltreter 2008), potencializando seu crescimento em diferentes microambientes da copa (Watkins et al. 2006), tais como os encontrados em florestas de altitudes elevadas.
Ressalta-se que, dentre as espécies citadas, duas são consideradas exóticas (Nephrolepis cordifolia e Pteris vittata), introduzidas e amplamente naturalizadas na América Tropical (Hovenkamp \& Miyamoto 2005; Prado \& Windisch 2000).

A análise da distribuição geográfica revelou a presença de cinco padrões, com predominância de espécies com distribuição Neotropical (57 espécies), corroborando com diversos outros estudos realizados no Brasil (e.g. Dittrich et al. 2005; Melo \& Salino 2007; Salino \& Almeida 2008; Matos 2009), sendo tal fato relacionado, principalmente, à capacidade de dispersão a longa distância das espécies de samambaias e licófitas. Treze espécies são restritas à América do Sul, 11 apresentam distribuição Pantropical e 16 são endêmicas do Brasil, sendo que, dessas, Cyathea abreviata apresenta distribuição restrita à região Nordeste (CE, PE, AL e BA) (Fernandes 2003).

\section{Similaridade florística}

Segundo o dendrograma obtido (Fig. 2), há a formação de dois grandes grupos: o primeiro grupo inclui todas as áreas de Floresta Amazônica, enquanto o segundo abrange principalmente áreas no Domínio da Floresta Atlântica, com algumas exceções.

No primeiro grupo é notável a baixa similaridade apresentada entre a REBIO Uatumã (UatumãAM) e as outras áreas, estas apresentando valores de similaridade mais altos entre si. Este resultado pode ser devido às características peculiares da reserva, a qual apresenta florestas sobre solos pedregosos e ricos em nutrientes (condição edáfica 
Tabela 2 - Lista das famílias e espécies de samambaias e licófitas da Serra da Jibóia, Santa Teresinha, BA, Brasil, com seus respectivos hábitos e distribuição geográfica. $\mathrm{E}=$ Epífita; $\mathrm{H}=$ Hemiepífita; $\mathrm{R}=\mathrm{Rupícola;} \mathrm{T}=\mathrm{Terrestre}$. $\mathrm{AS}=$ Distribuição limitada à América do Sul; BRA = Endêmica do Brasil; NEO = Distribuição ampla na região neotropical; $\mathrm{NO}=$ Distribuição restrita ao Nordeste do Brasil; PAN = Distribuição pantropical.

Table 2 - List of families and species of ferns and lycophytes of the Serra da Jibóia, Santa Teresinha, Bahia State, Brazil, with their habits and geographical distribution. $\mathrm{E}=$ Epiphyte; $\mathrm{H}=$ Hemiepiphyte; $\mathrm{R}=$ Lythophyte; $\mathrm{T}=$ Terrestrial. $\mathrm{AS}=$ Distribution limited to South America; BRA = Endemic of Brazil; $\mathrm{NEO}=$ Widely distributed in the neotropics; $\mathrm{NO}=$ Distribution restricted to Northeastern Brazil; PAN = Pantropical distribution.

\begin{tabular}{|c|c|c|c|c|}
\hline Famílias & Espécies & Hábito & $\begin{array}{c}\text { Distribuição } \\
\text { Geográfica }\end{array}$ & Voucher* \\
\hline \multirow[t]{4}{*}{ Anemiaceae } & Anemia hirta L. (Sw.) & $\mathrm{T} / \mathrm{R}$ & NEO & TSM 127 \\
\hline & Anemia mandiocana Raddi & $\mathrm{R}$ & BRA & TSM 142 \\
\hline & Anemia phyllitidis L. (Sw.) & $\mathrm{T}$ & NEO & TSM 109 \\
\hline & Anemia villosa Humb. \& Bonpl. ex Willd. & $\mathrm{T} / \mathrm{R}$ & AS & TSM 74 \\
\hline \multirow[t]{3}{*}{ Aspleniaceae } & Asplenium auriculatum $\mathrm{Sw}$. & $\mathrm{E}$ & NEO & TSM 90 \\
\hline & Asplenium feei Kunze ex Fée & $\mathrm{E}$ & NEO & TSM 213 \\
\hline & Asplenium serratum $\mathrm{L}$. & $\mathrm{E}$ & NEO & TSM 194 \\
\hline \multirow[t]{3}{*}{ Blechnaceae } & Blechnum brasiliense Desv. & $\mathrm{T}$ & NEO & TSM 137 \\
\hline & Blechnum occidentale $\mathrm{L}$. & $\mathrm{T}$ & NEO & TSM 188 \\
\hline & Salpichlaena volubilis (Kaulf.) J.Sm. & $\mathrm{T}$ & NEO & TSM 144 \\
\hline \multirow[t]{5}{*}{ Cyatheaceae } & Cyathea abreviata Fernandes & $\mathrm{T}$ & NO & TSM 161 \\
\hline & Cyathea corcovadensis (Raddi) Domin & $\mathrm{T}$ & BRA & RH 5860 \\
\hline & Cyathea delgadii Sternb. & $\mathrm{T}$ & NEO & TSM 113 \\
\hline & Cyathea nigra Linden ex Fournet & $\mathrm{T}$ & AS & TSM 224 \\
\hline & Cyathea phalerata Mart. & $\mathrm{T}$ & BRA & TSM 192 \\
\hline \multirow[t]{2}{*}{ Dennstaedtiaceae } & Dennstaedtia dissecta (Sw.) T.Moore & $\mathrm{T}$ & NEO & FRN 804 \\
\hline & Pteridium arachnoideum (Kaulf.) Maxon & $\mathrm{T}$ & NEO & TSM 88 \\
\hline \multirow[t]{17}{*}{ Dryopteridaceae } & Ctenitis distans (Brack.) Ching & $\mathrm{T}$ & BRA & TSM 87 \\
\hline & Ctenitis falciculata (Raddi) Ching & $\mathrm{T}$ & AS & LRN 3745 \\
\hline & Cyclodium meniscioides (Willd.) C.Presl & $\mathrm{T}$ & NEO & TSM 136 \\
\hline & Didymochlaena truncatula (Sw.) J.Sm. & $\mathrm{T}$ & PAN & TSM 149 \\
\hline & Elaphoglossum glabellum J.Sm. & E & NEO & TSM 221 \\
\hline & Elaphoglossum iguapense Brade & E & BRA & TSM 170 \\
\hline & Elaphoglossum insigne (Fée) Brade & E & BRA & TSM 134 \\
\hline & Elaphoglossum itatiayense Rosenst. & $\mathrm{E}$ & BRA & TSM 204 \\
\hline & Elaphoglossum lingua Brack. & E & NEO & TSM 219 \\
\hline & Elaphoglossum luridum (Fée) Christ & $\mathrm{E} / \mathrm{R} / \mathrm{T}$ & NEO & TSM 222 \\
\hline & Elaphoglossum macrophyllum (Mett.) Christ & $\mathrm{E}$ & AS & TSM 223 \\
\hline & Elaphoglossum pachydermum (Fée) Moore & $\mathrm{E} / \mathrm{R} / \mathrm{T}$ & BRA & TSM 211 \\
\hline & Elaphoglossum vagans (Mett.) Hieron. & $\mathrm{E}$ & BRA & TSM 220 \\
\hline & Mickelia guianensis (Aubl.) R.C. Moran & $\mathrm{H}$ & NEO & DC 2413 \\
\hline & Olfersia cervina (L.) Kunze & $\mathrm{T}$ & NEO & TSM 154 \\
\hline & Polybotrya speciosa Schott & $\mathrm{H}$ & BRA & TSM 201 \\
\hline & Rumohra adiantiformis (G. Forst.) Ching & $\mathrm{E}$ & PAN & TSM 196 \\
\hline \multirow[t]{3}{*}{ Gleicheniaceae } & Dicranopteris flexuosa (Schrad.) Underw. & $\mathrm{T}$ & NEO & TSM 124 \\
\hline & Gleichenella pectinata (Willd.) Ching & $\mathrm{T}$ & NEO & TSM 123 \\
\hline & Sticherus bifidus (Willd.) Ching & $\mathrm{T}$ & NEO & TSM 130 \\
\hline
\end{tabular}




\begin{tabular}{|c|c|c|c|c|}
\hline Famílias & Espécies & Hábito & $\begin{array}{l}\text { Distribuição } \\
\text { Geográfica }\end{array}$ & Voucher* \\
\hline \multirow[t]{10}{*}{ Hymenophyllaceae } & Didymoglossum krausii (Hook. \& Grev.) C.Presl & E & NEO & TSM 216 \\
\hline & Hymenophyllum asplenioides (Sw.) Sw. & $\mathrm{E}$ & NEO & TSM 199 \\
\hline & Hymenophyllum hirsutum (L.) Sw. & $\mathrm{E}$ & NEO & TSM 145 \\
\hline & Hymenophyllum polyanthos (Sw.) Sw. & $\mathrm{E}$ & PAN & TSM 115 \\
\hline & Polyphlebium diaphanum (Kunth) Ebihara \& Dubuisson & $\mathrm{E}$ & NEO & TSM 198 \\
\hline & Polyphlebium hymenophylloides (Bosch) Ebihara \& Dubuisson & $\mathrm{E}$ & NEO & TSM 217 \\
\hline & Trichomanes arbuscula Desv. & $\mathrm{E}$ & NEO & FRN 1077 \\
\hline & Trichomanes elegans Rich. & $\mathrm{E}$ & NEO & FRN 1076 \\
\hline & Trichomanes pilosum Raddi & $\mathrm{E}$ & AS & TSM 185 \\
\hline & Trichomanes polypodioides $\mathrm{L}$. & $\mathrm{E}$ & NEO & TSM 152 \\
\hline \multirow[t]{3}{*}{ Lindsaeaceae } & Lindsaea lancea (L.) Bedd. & $\mathrm{T} / \mathrm{R}$ & NEO & TSM 141 \\
\hline & Lindsaea quadrangularis Raddi & $\mathrm{T} / \mathrm{R}$ & AS & TSM 179 \\
\hline & Lindsaea stricta (Sw.) Dryand. & $\mathrm{T}$ & NEO & TSM 187 \\
\hline \multirow[t]{4}{*}{ Lomariopsidaceae } & Lomariopsis marginata (Schrad.) Kuhn in Decken & $\mathrm{H}$ & BRA & TSM 209 \\
\hline & Nephrolepis cordifolia (L.) C.Presl & $\mathrm{E}$ & PAN & TSM 200 \\
\hline & Nephrolepis pendula (Raddi) J.Sm. & $\mathrm{E}$ & NEO & TSM 197 \\
\hline & Nephrolepis rivularis (Vahl) Mett. ex Krug in Urban & E & NEO & TSM 176 \\
\hline \multirow[t]{3}{*}{ Lycopodiaceae } & Huperzia acerosa (Sw.) Holub & $\mathrm{E}$ & NEO & TSM 190 \\
\hline & Huperzia pungentifolia (Silveira) B.Øllg. & $\mathrm{T}$ & BRA & LRN 3319 \\
\hline & Lycopodiella cernua (L.) Pic.Serm. & $\mathrm{T}$ & PAN & TSM 112 \\
\hline Lygodiaceae & Lygodium volubile Sw. & $\mathrm{T}$ & NEO & TSM 139 \\
\hline Marattiaceae & Danaea geniculata Raddi & $\mathrm{T}$ & BRA & TSM 210 \\
\hline Ophioglossaceae & Ophioglossum palmatum L. & $\mathrm{E}$ & PAN & TSM 173 \\
\hline \multirow[t]{16}{*}{ Polypodiaceae } & Campyloneurum acrocarpon Fée & $\mathrm{E}$ & BRA & TSM 225 \\
\hline & Campyloneurum nitidum (Kaulf.) C.Presl & $\mathrm{E}$ & AS & TSM 205 \\
\hline & Cochlidium serrulatum (Sw.) L.E.Bishop & $\mathrm{E} / \mathrm{R}$ & PAN & TSM 86 \\
\hline & Dicranoglossum furcatum (L.) J.Sm. & $\mathrm{E}$ & NEO & TSM 143 \\
\hline & Lellingeria suspensa (L.) A.R.Sm. \& R.C.Moran & $\mathrm{E}$ & NEO & TSM 189 \\
\hline & Melpomene melanosticta (Kunze) A.R.Sm. \& R.C.Moran & $\mathrm{R}$ & NEO & TSM 118 \\
\hline & Microgramma lycopodioides (L.) Copel. & $\mathrm{E}$ & NEO & TSM 184 \\
\hline & Microgramma reptans (Cav.) A.R.Sm. & $\mathrm{E}$ & NEO & TSM 93 \\
\hline & Microgramma vacciniifolia (Langsd. \& Fisch.) Copel. & $\mathrm{E}$ & NEO & TSM 207 \\
\hline & Pecluma pilosa (A.M.Evans) M.Kessler \& A.R.Sm. & $\mathrm{E}$ & NEO & TSM 191 \\
\hline & Pecluma sicca (Lindm.) M.G.Price & $\mathrm{E}$ & AS & TSM 206 \\
\hline & Phlebodium areolatum (Humb. \& Bonpl. ex Willd.) J.Sm. & $\mathrm{E}$ & NEO & TSM 114 \\
\hline & Pleopeltis astrolepis (Liebm.) E.Fourn. & $\mathrm{E}$ & NEO & TSM 174 \\
\hline & Pleopeltis macrocarpa (Bory ex Willd.) Kaulf. & $\mathrm{E}$ & PAN & TSM 175 \\
\hline & Serpocaulon catharinae (Langsd. \& Fisch.) A.R.Sm. & $\mathrm{E}$ & AS & TSM 75 \\
\hline & Serpocaulon triseriale (Sw.) A.R.Sm. & $\mathrm{E} / \mathrm{T}$ & NEO & TSM 195 \\
\hline Psilotaceae & Psilotum nudum (L.) P.Beauv. & $\mathrm{E}$ & PAN & TSM 202 \\
\hline \multirow[t]{3}{*}{ Pteridaceae } & Adiantopsis radiata $(\mathrm{L}$.$) Fée$ & $\mathrm{T}$ & NEO & TSM 129 \\
\hline & Adiantum abscissum Schrad. & $\mathrm{T}$ & AS & TSM 138 \\
\hline & Adiantum deflectens Mart. & $\mathrm{T}$ & NEO & FRN 807 \\
\hline
\end{tabular}




\begin{tabular}{|c|c|c|c|c|}
\hline Famílias & Espécies & Hábito & $\begin{array}{l}\text { Distribuição } \\
\text { Geográfica }\end{array}$ & Voucher* \\
\hline & Adiantum lucidum (Cav.) Sw. & $\mathrm{T}$ & $\mathrm{NEO}$ & TSM 166 \\
\hline & Adiantum tetraphyllum Humb. \& Bonpl. ex Willd. & $\mathrm{T}$ & $\mathrm{NEO}$ & TSM 167 \\
\hline & Doryopteris collina (Raddi) J.Sm. & $\mathrm{T}$ & AS & TSM 176 \\
\hline & Doryopteris pentagona Pic.Serm. & $\mathrm{E}$ & AS & FRN 810 \\
\hline & Pteris decurrens C.Presl & $\mathrm{E}$ & AS & TSM 150 \\
\hline & Pteris denticulata Sw. & $\mathrm{E}$ & $\mathrm{NEO}$ & TSM 110 \\
\hline & Pteris vittata $\mathrm{L}$. & $\mathrm{R}$ & PAN & TSM 99 \\
\hline & Vittaria graminifolia Kaulf. & $\mathrm{E}$ & $\mathrm{NEO}$ & TSM 95 \\
\hline & Vittaria lineata (L.) Sm. & $\mathrm{E}$ & $\mathrm{NEO}$ & TSM 169 \\
\hline Saccolomataceae & Saccoloma elegans Kaulf. & $\mathrm{T}$ & $\mathrm{NEO}$ & TSM 153 \\
\hline Schizaeaceae & Schizaea elegans (Vahl) Sw. & $\mathrm{T}$ & $\mathrm{NEO}$ & TSM 183 \\
\hline \multirow[t]{2}{*}{ Selaginellaceae } & Selaginella flexuosa Spring & $\mathrm{T}$ & $\mathrm{NEO}$ & TSM 104 \\
\hline & Selaginella muscosa Spring & $\mathrm{T} / \mathrm{R}$ & $\mathrm{NEO}$ & TSM 103 \\
\hline Tectariaceae & Triplophyllum hirsutum (Holttum) J.Prado \& R.C.Moran & $\mathrm{T}$ & $\mathrm{NEO}$ & TSM 212 \\
\hline \multirow[t]{3}{*}{ Thelypteridaceae } & Thelypteris conspersa (Schrad.) A.R.Sm. & $\mathrm{T}$ & $\mathrm{NEO}$ & FRN 1060 \\
\hline & Thelypteris hispidula (Decne.) C.F.Reed & $\mathrm{T}$ & PAN & TSM 185 \\
\hline & Thelypteris villosa (Link) C.F.Reed & $\mathrm{T}$ & BRA & TSM 168 \\
\hline
\end{tabular}

Dice-Sorensen

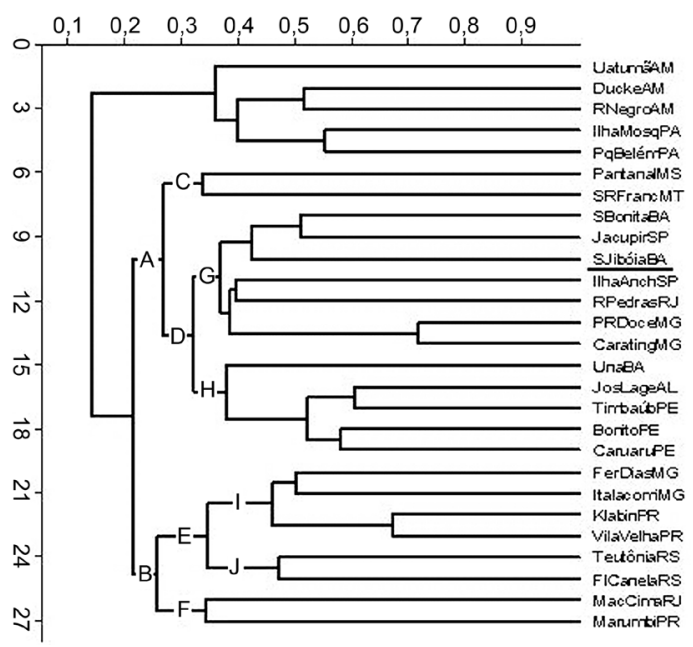

Figura 2 - Dendrograma de similaridade florística para sambaias e licófitas, entre a Serra da jibóia (sublinhada) e outras 26 áreas brasileiras, obtido através do algoritmo de Sørensen e análise de agrupamento UPGMA. As áreas aparecem em formato de códigos especificados na Tabela 1.

Figure 2 - Dendrogram of floristic similarity for fens and lycophytes among Serra da Jibóia (underlined) and 25 other brazilian areas, obtained through the algorithm of Sørensen and UPGMA cluster analysis. The area codes are specified in Table 1. rara na Amazônia Central) que, segundo Zuquim et al. (2008), abriga a maior diversidade de samambaias e licófitas já registradas para a Amazônia Central.

No segundo grupo nota-se a formação de dois outros grupos menores, A e B. Tais grupos parecem, de certa forma, arranjados através de uma variação latitudinal, havendo uma separação discreta entre áreas do norte e sul do Brasil.

No grupo A observa-se claramente a separação de um ramo (C) constituído pelo Pantanal-MS (PantanalMS) e Serra Ricardo Franco-MT (SRFrancMT). Tal situação era de certa forma esperada, já que nestas áreas a flora de samambaias e licófitas apresenta espécies típicas do Cerrado do Brasil Central e ainda sofre influência de elementos andinos, bem como oriundos do Chaco boliviano, paraguaio e argentino (Assis 2007; Windisch \& Tryon 2001). Apesar de tal separação, estas duas áreas ainda mostram uma relação florística com o grupo D (que abrange áreas de Floresta Atlântica). Esta relação pode ser explicada pela presença de espécies com ampla distribuição geográfica, já que como o coeficiente de Sørensen, assim como o de Jaccard, agrupa pela dupla presença, a existência de tais espécies poderia estar agrupando essas áreas distintas. 
O grupo D reúne áreas de Floresta Atlântica situadas mais ao norte do país, quando comparadas com as demais do grupo B, que agrega as áreas localizadas mais ao sul (com algumas exceções); ambos compostos tanto de floresta ombrófila quanto de floresta semidecídua. Estes resultados corroboram com as afirmações de Oliveira-Filho $\&$ Ratter (1995), Oliveira-Filho \& Fontes (2000) e Oliveira-Filho et al. (2005), em seus trabalhos com angiospermas. Segundo estes autores, as florestas ombrófilas e semidecíduas fazem parte de um mesmo contínuo com forte variação latitudinal e, apesar das diferenças florísticas entre as duas fisionomias, as florestas ombrófilas localizadas mais ao norte estão mais próximas das suas vizinhas semidecíduas do norte do que das florestas ombrófilas localizadas mais ao sul.

Ainda no grupo D, observa-se que este é composto por todas as áreas localizadas na Região Nordeste e algumas da Região Sudeste do Brasil. O grupo apresenta uma dicotomia, na qual o primeiro ramo $(\mathrm{G})$ apresenta um subgrupo com a Serra Bonita (SBonitaBA), localizada no sul da Bahia, mostrando uma similaridade de 0,5 com o Parque Estadual do Jacupiranga (JacupirSP), localizado ao sul do estado de São Paulo, além da Serra da Jibóia (SJibóiaBA) aparecer relacionada a estas duas áreas com similaridade de 0,4 . Tais resultados sugerem que, apesar de localidades próximas tenderem a apresentar maior similaridade, as condições ambientais possivelmente exercem grande influência na composição florística de samambaias e licófitas de uma região.

A distribuição de samambaias é mais fortemente determinada pela disponibilidade de habitat do que pela sua elevada capacidade de dispersão (Tryon 1970; Smith 1972, 1993; Tuomisto et al. 2003; Moran 2008; Kessler 2010). As relações de afinidade entre estas áreas, apesar de não serem elevadas, sugerem que a Serra da Jibóia apresenta condições ambientais semelhantes às outras duas. A vegetação da vertente oriental da serra recebe influência das chuvas orográficas (chuvas de relevo), onde os ventos úmidos provindos do litoral, ao encontrar essa barreira, fazem precipitar grande parte da umidade. A porção ocidental, por conseguinte, é menos úmida e caracterizada por Floresta Estacional (Sandes, 2003), além da formação de campo rupestre no topo. Segundo Fernandes (2000), as matas úmidas, disjunções florestais circundadas pelas caatingas, representam, pela similaridade vegetacional e florística, remanescentes de mata atlântica. Tais disjunções podem ser explicadas dada a existência local de fatores de exceção, pela origem climática, topografia, hidrologia ou mesmo litologia, ligados a registros paleontológicos. Além disso, é importante destacar que as três áreas do referido subgrupo correspondem a regiões com elevadas altitudes, o que proporciona, nestas regiões relativamente distantes geograficamente, grande diversidade de condições físicas, climáticas e edáficas, aumentando a heterogeneidade ambiental e a disponibilidade de diferentes hábitats.

A afinidade apresentada entre estas três áreas e destas com o outro subgrupo que engloba áreas da Região Sudeste ainda permite inferir que a flora de samambaias e licófitas da Bahia está mais relacionada ao Sudeste do que ao restante da Região Nordeste. Tal semelhança também é citada por Matos et al. (2010) o qual encontrou na Serra Bonita (sul da Bahia) um número significativo de espécies características das regiões montanhosas da Floresta Atlântica do Sudeste do Brasil, sendo esta relação refletida no dendrograma (Fig. 2) com a união da referida área com o Parque Estadual do Jacupiranga. Além disso, a considerável relação com áreas do Sudeste do Brasil é referida em outros trabalhos realizados na Serra da Jibóia com diferentes grupos de organismos, tais como Valente \& Porto (2006) com hepáticas, Sobrinho \& Queiroz (2005) com angiospermas e Juncá (2006) com anuros. Contudo, vale destacar que os levantamentos florísticos para samambaias e licófitas no estado ainda são escassos, principalmente no que diz respeito aqueles realizados no domínio da Floresta Atlântica, sendo possível a mudança de tais relações à medida que novos inventários sejam realizados.

$\mathrm{O}$ outro ramo do grupo $\mathrm{G}$ abriga áreas do sudeste, conforme mencionado anteriormente. Duas áreas litorâneas aparecem relacionadas: a Reserva Rio das Pedras (RPedrasRJ), localizada região sul do RJ com o Parque Estadual da Ilha Anchieta (IlhaAnchSP), ao norte de SP, e estas duas, juntamente com as áreas da Bacia do Rio Doce - MG (com similaridade de 0,7), compõem subgrupo G. A elevada similaridade destas duas últimas áreas era esperada devido à proximidade geográfica das mesmas.

No conjunto $\mathrm{H}$ observa-se o agrupamento da REBIO Una (UnaBA) com os Brejos de Altitude nordestinos. Apesar da referida reserva estar localizada na região do tabuleiro sul baiano, esta não formou um agrupamento com as outras 
áreas localizadas no estado e nem com áreas do sudeste, como visto para a Serra Bonita e Serra da Jibóia, mas sim com as regiões dos brejos de altitude de Alagoas e Pernambuco, distantes geograficamente da REBIO. Resultados semelhantes foram encontrados por Matos (2009), o qual atribuiu tal fato às condições de umidade nas áreas mais baixas do sul da Bahia que podem ser comparáveis às encontradas nos brejos de altitude nordestinos. Estes, apesar de estarem inseridos na vegetação de caatinga, localizam-se em terrenos elevados que apresentam um clima mais ameno, com melhores condições de umidade em relação ao restante da vegetação circundante.

O grupo B, composto basicamente pelas áreas de Floresta Atlântica do sul do país, apresenta uma dicotomia, onde o primeiro ramo (E) abriga áreas de florestas estacionais e/ou ombrófilas mistas. Este apresenta um subgrupo (I) que agrega áreas de Minas Gerais (FerDiasMG e ItalacomiMG) e do Paraná (KlabinPR e VilaVelhaPR), estas últimas apresentando elevada similaridade (ca. $0,7)$ e outro subgrupo $(\mathrm{J})$ com as duas áreas localizadas no Rio Grande do Sul (TeutôniaRS e FlCanelaRS). O ramo F, por conseguinte, inclui duas áreas de floresta ombrófila densa, distantes geograficamente (Reserva Ecológica de Macaé de Cima - RJ e Parque Estadual Pico do Marumbi - PR), unidas talvez por influência do perfil altitudinal dessas regiões que pode conferir características ambientais semelhantes.

Os resultados demonstram que a Serra da Jibóia, apesar de estar inserida no semiárido baiano, apresenta características típicas das matas úmidas de altitude, com riqueza florística e diversidade de hábitos e hábitats. Tais características singulares da área podem ser explicadas principalmente pela topografia do terreno representada por um maciço de morros inseridos em uma área de caatinga de relevo plano. O Morro da Pioneira apresenta relevo acidentado com altitudes de até $800 \mathrm{~m}$, fato que favorece a presença de áreas mais úmidas pela influência das chuvas orográficas, favorecendo o surgimento e desenvolvimento de samambaias e licófitas. Neste contexto, o presente estudo sugere uma maior similaridade florística das samambaias e licófitas da região com o sul da Bahia e Sudeste do Brasil, reforçando as peculiaridades deste remanescente de mata atlântica.

\section{Agradecimentos}

O primeiro autor agradece aos seus familiares, a ajuda durante o trabalho de campo e ao $\mathrm{CNPq}$, a bolsa de Mestrado concedida. Agradecemos também aos curadores dos herbários visitados, ao PPGBot-UEFS, ao HUEFS e seus funcionários, a logística e infra-estrutura fornecida durante a realização do trabalho.

\section{Referências}

Assis, E.L.M. 2007. Pteridófitas da borda oeste do Pantanal sul-Matogrossense, Brasil. Dissertação de Mestrado. Universidade Federal do Paraná, Setor de Ciências Biológicas, Curitiba. 187p.

Athayde Filho, F.P.; Pereira, V.S.; Smidt, E.C. \& Nonato, F.R. 2003. Pteridófitas do Parque Estadual da Ilha Anchieta (PEIA), Ubatuba, São Paulo, Brasil. Bradea 9: 55-66.

Barros, I.C.L. 1998. Pteridófitas. In: Guedes, M.L.S. \& Orge, M.D. (eds.). Checklist das espécies vasculares do Morro do Pai Inácio (Palmeiras) e Serra da Chapadinha (Lençóis) Chapada Diamantina. Bahia - Brasil. Universidade Federal da Bahia, Salvador. Pp. 12, 35-36.

Barros, I.C.L. \& Araújo, S.S. 2000. Flora pteridofítica do Rio Mosquito, município de Lençóis, Chapada Diamantina Bahia, Brasil. Notas Preliminares. Boletim da Sociedade Broteriana 1: 145-162.

Benzing, D.H. 1990. Vascular Epiphytes: general biology and related biota. Cambridge University Press, Cambridge. $376 \mathrm{p}$.

Conservação Internacional do Brasil; Fundação SOS Mata Atlântica; Fundação Biodiversitas; Instituto de Pesquisas Ecológicas; Secretaria do Meio Ambiente do Estado de São Paulo \& Instituto Estadual de Florestas-MG. 2000. Avaliação e ações prioritárias para a conservação da biodiversidade da Mata Atlântica e Campos Sulinos. Ministério do Meio Ambiente/SBF, Brasília. 40p.

Costa, J.F. \& Pietrobom, M.R. 2007. Pteridófitas (Lycophyta e Monilophyta) da Ilha de Mosqueiro, município de Belém, estado do Pará, Brasil. Boletim do Museu Paraense Emílio Goeldi, Ciências Naturais 2: 45-55.

Costa, J.F.; Souza, M.G.C \& Pietrobom, M.R. 2006. Levantamento florístico das Pteridófitas (Lycophyta e Monilophyta) do Parque Ambiental de Belém (Belém, Pará, Brasil). Revista de Biologia Neotropical 3: 4-12.

Costa, M.A.S.; Prado, J.; Windisch, P.G.; Labiak, P.H. \& Freitas, C.A.A. 1999. Pteridophyta. In: Ribeiro, J.E.L.S.; Hopkins, M.J.G.; Vicentini, A.; Sothers, C.A.; Costa, M.A.S.; Brito, J.M.; Souza, M.A.D.; Martins, L.H.P.; Lohmann, L.G.; Assunção, P.A.C.L.; Pereira, E.C.; Silva, C.F.; Mesquita, M.R. 
\& Procópio, L.C. (eds.). Flora da Reserva Ducke. Guia de identificação das plantas vasculares de uma mata de terra-firme na Amazônia Central. INPA/ DIFD, Manaus. Pp. 97-117.

Dittrich, V.A.; Waechter, J.L. \& Salino, A. 2005. Species richness of pteridophytes in a montane Atlantic rain forest plot of Southern Brazil. Acta Botanica Brasilica 19: 519-525.

Edwards, P.J. 1995. Ferns. In: Stannard, B.L. (ed.). Flora of the Pico das Almas, Chapada Diamantina - Bahia, Brasil. Royal Botanic Gardens, Kew. Pp. 83-84.

Fernandes, A. 2000. Fitogeografia brasileira. $2^{\text {a }}$ ed. Multigraf, Fortaleza. 340p.

Fernandes, I. 2003. Taxonomia dos representantes de Cyatheaceae do nordeste oriental do Brasil. Pesquisas Botânica 53: 7-53.

Ferreira, J.L.; Melo, E. \& Nonato, F.R.2012. Schizaeales da Chapada Diamantina, Bahia, Brasil. Rodriguésia 63: 451-461.

Freitas, C.A.A. \& Prado, J. 2005. Lista anotada das pteridófitas de florestas inundáveis do alto Rio Negro, município de Santa Isabel do Rio Negro, AM, Brasil. Acta Botanica Brasilica 19: 399-406.

Hammer, Ø.; Harper, D.A.T. \& Ryan, D.T. 2001. PAST: palaeontological statistics software package for education and data analysis. Palaeontologia Electronica 4: 1-9.

Hovenkamp, P.H. \& Miyamoto, F. 2005. A conspectus of the native and naturalized species of Nephrolepis (Nephrolepidaceae) in the world. Blumea 50: 279322.

Juncá, F.A. 2006. Diversidade e uso de hábitat por anfíbios anuros em duas localidades de Mata Atlântica, no norte do estado da Bahia. Biota Neotropica 6: 1-17.

Kessler, M. 2010. Biogeography of ferns. In: Mehltreter, K.; Walker, L.R. \& Sharpe, J.M. (eds). Fern ecology. Cambridge University Press, New York. Pp. 22-60

Kornaš, J. 1977. Life-forms and seasonal patterns in the pteridophytes in Zambia. Acta Societatis Botanicorum Poloniae 46: 669-690.

Kramer, K.U. \& P.S.Green. 1990. Pteridophytes and Gymnosperms. In: Kubitzki, K. (ed.). The families and genera of vascular plants. Springer-Verlag, Berlin. Pp. 1-404.

Labiak, P.H. \& Prado, J. 1998. Pteridófitas epífitas da Reserva Volta Velha, Itapoá, Santa Catarina, Brasil. Boletim do Instituto de Botânica 11: 1-79.

Lima, H.C.; Morim, M.P.; Guedes-Bruni, R.R.; Sylvestre, L.S.; Pessoa, S.V.A.; Silva Neto, S. \& Quinet, A. 2001. Reserva ecológica de Macaé de Cima, Nova Friburgo, Rio de Janeiro: Lista das espécies vasculares. Disponível em <http://www. jbrj.gov.br/pesquisa/pma/macaedecima/checklist/ Lista_Plantas_Vasculares.htm>. Acesso em 19 Set 2010 .
Lima, M.P.M.; Guedes-Bruni, R.R.; Sylvestre, L.S.; Pessoa, S.A.V. \& Andreata, R.H.P. 1997. Padrões de distribuição geográfica das espécies vasculares da Reserva Ecológica de Macaé de Cima. In: Lima, H.C. \& Guedes-Bruni, R.R. (eds.). Serra de Macaé de Cima: diversidade florística e conservação em Mata Atlântica. Jardim Botânico do Rio de Janeiro, Rio de Janeiro. Pp. 103-122.

Macedo, T.S.; Góes-Neto, A. \& Nonato, F.R. 2011. Primeira ocorrência de Thelypteris villosa (Link) C. F. Reed (Thelypteridaceae) para o Nordeste do Brasil. Acta Botanica Brasilica 25: 727-728.

Macedo, T.S.; Góes-Neto, A. \& Nonato, F.R. 2012. Samambaias e licófitas de um fragmento de mata atlântica na Serra da Jibóia, município de Santa Teresinha, Bahia, Brasil. Sitientibus Série Ciências Biológicas 12. Disponível em $<$ http://periodicos. uefs.br/ojs/index.php/sitientibusBiologia/article/ view/98/115>.

Margurran, A.E. 1988. Ecological diversity and its measurement. Princeten University Press, Princeton. $192 \mathrm{p}$.

Matos, F.B. 2009. Samambaias e licófitas da RPPN Serra Bonita, Município de Camacan, Sul da Bahia, Brasil. Dissertação de Mestrado. Universidade Federal do Paraná, Curitiba. 237p.

Matos, F.B.; Amorim, A.M. \& Labiak, P.H. 2010. The ferns and lycophytes of a Montane Tropical Forest in Southern Bahia, Brazil. Journal of the Botanical Research Institute of Texas 4: 1-14.

Mehltreter, K. 2008. Phenology and habitat specificity of tropical ferns. In: Ranker, T.A. \& Haufler, C.H. (eds.). Biology and evolution of ferns and Lycophytes. Cambridge University Press, New York. Pp 201-221.

Melo, L.C.N. \& Salino, A. 2002. Pteridófitas de duas áreas de floresta da bacia do Rio Doce no Estado de Minas Gerais, Brasil. Lundiana 3: 129-139.

Melo, L.C.N. \& Salino, A. 2007. Pteridófitas em fragmentos florestais da APA Fernão Dias, Minas Gerais. Rodriguésia 58: 207-220.

Mittermeier, R.A.; Gil, P.R.; Hoffmann, M.; Pilgrim, J.; Brooks, T.; Mittermeier, C.G.; Lamourex, J. \& Fonseca, G.A.B. 2004. Hotspots revisited. CEMEX, Mexico City. 392p.

Moran, R.C. 2008. Diversity, biogeography, and floristics. In: Ranker, T.A. \& Haufler, C.H. (eds.). Biology and evolution of ferns and lycophytes. Cambridge University Press, New York. Pp. 201221.

Moran, R.C.; Klimas, S. \& Carlsen, M. 2003. Low-trunk epiphytic ferns on tree ferns versus angiosperms in Costa Rica. Biotropica 35: 48-56.

Mori, S.A.; Boom, B.M.; Carvalho, A.M. \& Santos, T.S. 1983. Southern Bahian Moist Forests. Botanical Review 49: 1-155. 
Myers, N.; Mittermeier, R.A.; Mittermeier, C.G.; Fonseca, G.A.B. \& Kent, J. 2000. Biodiversity hotspots for conservation priorities. Nature 403: 853-845.

Mynssen, C.M. \& Windisch, P.G. 2004. Pteridófitas da Reserva Rio das Pedras, Mangaratiba, RJ, Brasil. Rodriguésia 55: 125-156.

Noblick, L.R. \& Brito, I.C. 1984. Pteridófitas dos arredores da Vila de Lamarão do Passé, São Sebastião do Passé, Bahia. Sitientibus 2: 17-28.

Nonato, F.R. 2005. Pteridófitas. In: Juncá, F.A.; Funch, L. \& Rocha, W. (eds.). Biodiversidade e conservação da Chapada Diamantina. Ministério do Meio Ambiente, Brasília. Pp. 211-223.

Øllgaard, B. 1995. Lycopodiaceae. In: Stannard, B.L. (ed.). Flora of the Pico das Almas, Chapada Diamantina-Bahia, Brasil. Royal Botanic Gardens, Kew. Pp. 80-83.

Oliveira-Filho, A.T. \& Fontes, A.L. 2000. Patterns of floristic differentiation among Atlantic Forests in Southeastern Brazil and the influence of climate. Biotropica 32: 793-810.

Oliveira-Filho, A.T. \& Ratter, J.A. 1995. A study of the origin of central Brazilian forests by the analysis of plant species distribution patterns. Edinburgh Journal of Botany 52: 141-194.

Oliveira-Filho, A.T.; Tameirão-Neto, E.; Carvalho, W.A.C.; Werneck, M.; Brina, A.E.; Vidal, C.V.; Resende, S.C. \& Pereira, J.A.A. 2005. Análise florística do compartimento arbóreo de áreas de Floresta Atlântica sensu lato na região das Bacias do Leste (Bahia, Minas Gerais, Espírito Santo e Rio de Janeiro). Rodriguésia 56: 185-235.

Paciência, M.L.B. 2008. Diversidade de pteridófitas em gradientes de altitude na mata atlântica do estado do Paraná, Brasil. Tese de Doutorado. Universidade de São Paulo, São Paulo. 229f.

Paciência, M.L.B. \& Prado, J. 2004. Efeitos de borda sobre a comunidade de pteridófitas na Mata Atlântica na região de Una, sul da Bahia, Brasil. Revista Brasileira de Botânica 27: 641-653.

Paciência, M.L.B. \& Prado, J. 2005. Distribuição da assembléia de pteridófitas em uma paisagem fragmentada de Mata Atlântica, no sul da Bahia, Brasil. Hoehnea 32: 103-117.

Pichi-Sermolli, R.E.G. 1996. Authors of scientific names in Pteridophyta. Royal Botanical Gardens, Kew. 78p.

Pietrobom, M.R. \& Barros, I.C.L. 2006. Associações entre as espécies de pteridófitas em dois fragmentos de Floresta Atlântica do nordeste brasileiro. Biotemas 19: 15-26.

Pietrobom, M.R. \& Barros, I.C.L. 2007. Pteridoflora do Engenho Água Azul, município de Timbaúba, Pernambuco, Brasil. Rodriguésia 58: 85-94.
Prado, J. 1995. Ferns. In: Stannard, B.L. (ed.). Flora of the Pico das Almas, Chapada Diamantina-Bahia, Brasil. Royal Botanic Gardens, Kew. Pp. 85-110

Prado, J. 2003. Pteridófitas. In: Zappi D.C.; Lucas E.; Stannard, B.L.; Lughadha, E.N.; Pirani, J.R.; Queiroz, L.P.; Atkins, S.; Hind, D.J.N.; Giulietti, A.M.; Harley, R.M. \& Carvalho, A. M. Lista das plantas vasculares de Catolés, Chapada Diamantina, Bahia, Brasil. Boletim de Botânica da Universidade de São Paulo 21: 359-360.

Prado, J. \& Windisch, P.G. 2000. The genus Pteris L. (Pteridaceae) in Brazil. Boletim do Instituto de Botânica 13: 103-199.

Rolim, L.B. 2007. Pteridófitas do Parque Estadual do Italacomi, Minas Gerais, Brasil. Dissertação de Mestrado. Universidade de Brasília, Brasília. 288p.

Rouhan, G.; Dubuisson, J.Y.; Rakotondrainibe, F.; Motley, T.J.; Mickel, J.T.; Labat, J.N. \& Moran, R.C. 2004. Molecular phylogeny of the fern genus Elaphoglossum (Elaphoglossaceae) based on chloroplast non-coding DNA sequences: contributions of species from the Indian Ocean area. Molecular Phylogenetics and Evolution 33: 745-763.

Sakagami, C.R. 2006. Pteridófitas do parque ecológico da Klabin, Telêmaco Borba, Paraná, Brasil. Dissertação de Mestrado. Universidade Federal do Paraná, Curitiba. 200p.

Salino, A. \& Almeida, T.E. 2008. Pteridófitas do Parque Estadual do Jacupiranga, SP, Brasil. Acta Botanica Brasilica 22: 983-991.

Salino, A. \& Almeida, T.E. 2009. Pteridófitas. In: Stehmann, J.R.; Forzza, R.C.; Salino, A.; Sobral, M.; Costa, D.P. \& Kamino, L.H.Y. (eds.). Plantas da Floresta Atlântica. Jardim Botânico do Rio de Janeiro, Rio de Janeiro. Pp. 19-26.

Sandes, A.B. 2003. Releitura socioambiental da Serra da Jibóia: um estudo voltado para a produção continuada em Educação Ambiental. Monografia de Graduação. Universidade Estadual de Feira de Santana, Feira de Santana. 17p.

Santiago, A.C.P.; Barros, I.C.L. \& Sylvestre, L.S. 2004. Pteridófitas ocorrentes em três fragmentos florestais de um brejo de altitude (Bonito, Pernambuco, Brasil). Acta Botanica Brasílica 18: 781-792.

Schmitt, J.L.; Fleck R.; Burmeister, E.L. \& Rubio, M.A.K. 2006. Diversidade e formas biológicas de pteridófitas da Floresta Nacional de Canela, Rio Grande do Sul: contribuições para o plano de manejo. Pesquisas, Botânica 57: 275-288.

Schwartsburd, P.B. \& Labiak, P.H. 2007. Pteridófitas do Parque Estadual de Vila Velha, Ponta Grossa, Paraná, Brasil. Hoehnea 34: 159-209.

Smith, A.R. 1972. Comparison of fern and flowering plant distributions with some evolutionary interpretations for ferns. Biotropica 4: 4-9. 
Smith, A.R. 1993. Phytogeographic principles and their use in understanding fern relationships. Journal of Biogeography 20: 255-64.

Smith, A.R.; Pryer, K.M.; Schuettpelz, E.; Korall, P.; Schneider, H. \& Wolf, P.G. 2006. A classification for extant ferns. Taxon 55: 705-731.

Sobrinho, J.G.C. \& Queiroz, L.P. 2005. Composição florística de um fragmento de Mata Atlântica na Serra da Jibóia, Santa Terezinha, Bahia, Brasil. Sitientibus Série Ciências Biológicas 5: 20-28.

Steffens, C. \& Windisch, P.G. 2007. Diversidade e formas de vida de pteridófitas no Morro da Harmonia em Teutônia - RS, Brasil. Pesquisas, Botânica 58: 375-382.

Sylvestre, L.S. 1997. Pteridófitas da Reserva Ecológica de Macaé de Cima. In: Lima, H.C. \& Guedes-Bruni, R.R. (eds.). Serra de Macaé de Cima: diversidade florística e conservação em Mata Atlântica. Ministério do Meio Ambiente e da Amazônia Legal/ IBAMA. Jardim Botânico do Rio de Janeiro, Rio de Janeiro. Pp. 41-52.

Thiers, B. 2010. [continuously updated]. Index Herbariorum: A global directory of public herbaria and associated staff. New York Botanical Garden's Virtual Herbarium. Disponível em $<$ http://sweetgum.nybg.org/ih/>. Acesso em 7 Abr 2010.

Tryon, R.M. 1970. Development and evolution of fern floras of oceanic islands. Biotropica 2: 76-84.

Tuomisto, H.; Ruokolainen, K.; Aguilar, M. \& Sarmientos, A. 2003. Floristic patterns along a 43-km long transect in an Amazonian rain forest. Journal of Ecology 91: 743-56.

Valente, E.B. \& Porto, K.C. 2006. Hepáticas (Marchantiophyta) de um fragmento de Mata Atlântica na Serra da Jibóia, município de Santa Teresinha, BA, Brasil. Acta Botanica Brasilica 20: 433-441.

Veloso, H.P.; Rangel-Filho, A.L.R. \& Lima, J.C.A. 1991. Classificação da vegetação brasileira, adaptada a um sistema universal. Fundação Instituto Brasileiro de Geografia e Estatística, Rio de Janeiro. 123p.

Xavier, S.R.S. \& Barros, I.C.L. 2005. Pteridoflora e seus aspectos ecológicos ocorrentes no Parque Estadual João Vasconcelos Sobrinho, Caruaru, PE, Brasil. Acta Botanica Brasilica 19: 775-781.

Watkins, J.E.Jr.; Cardelús, C.; Colwell, R.K. \& Moran, R.C. 2006. Species richness and distribution of ferns along an elevational gradient in Costa Rica. American Journal of Botany 93: 73-83.

Windisch, P.G. 1992. Pteridófitas da região Norteocidental do estado de São Paulo: guia para estudos e excursões. $2^{\text {a }}$ ed. Universidade Estadual Paulista, São José do Rio Preto. 110p.

Windisch P.G. \& Tryon, R.M. 2001. The Serra Ricardo Franco (State of Mato Grosso, Brazil) as probable migration route and its present fern flora. Bradea 8: 267-276.

Zuquim, G.; Costa, F.R.C.; Prado, J. \& Tuomisto, H. 2008. Guia de samambaias e licófitas da REBIO Uatumã - Amazônia Central. Attema Design Editorial, Manaus. 315p. 